\title{
Adesão a puericultura para o seguimento à saúde infantil
}

\author{
Adherence to childcare for child health follow-up \\ Adherencia al cuidado de niños para el seguimiento de la salud infantil
}

Recebido: 13/05/2021 | Revisado: 19/05/2021 | Aceito: 24/05/2021 | Publicado: 09/06/2021

Jaqueline Pereira Jornooki
ORCID: https://orcid.org/0000-0002-0828-5069
Universidade Estadual do Oeste do Paraná, Brasil
E-mail: jaquelinejornooki@ @mail.com
Ana Paula Contiero Toninato
ORCID: https://orcid.org/0000-0002-7251-6423
Universidade Estadual do Oeste do Paraná, Brasil
E-mail: ana_contiero@ hotmail.com
Helder Ferreira
ORCID: https://orcid.org/0000-0003-0715-8057
Universidade Estadual do Oeste do Paraná, Brasil
E-mail: heelfer@ @mail.com
Rosangela Aparecida Pimenta Ferrari
ORCID: https://orcid.org/0000-0003-0157-7461
Universidade Estadual de Londrina, Brasil
E-mail: ropimentaferrari@uel.br
Adriana Zilly
ORCID: https://orcid.org/0000-0002-8714-8205
E-mail: aazilly @ hotmail.com
Universidade Estadual do Oete do Paná, Brasil
Rosane Meire Munhak da Silva
ORCID: https://orcid.org/0000-0003-3355-0132
Universidade Estadual do Oeste do Paraná, Brasil
E-mail: zanem2010@ hotmail.com

\section{Resumo}

Objetivo: avaliar as ações desenvolvidas na puericultura e os fatores relacionados ao seguimento à saúde infantil. Método: pesquisa transversal, analítica, com coleta de dados entre 2017 e 2018, com primeiro contato no alojamento conjunto de maternidades, 24 horas após o parto; e nos domicílios após seis meses, com a participação de 280 mães. Para análise foi realizado o teste de associação Qui-Quadrado ou teste exato de Fischer, com nível de significância de $5 \%(\mathrm{p}<0,05)$. Resultados: a maioria das consultas de puericultura foi realizada por enfermeiros, de forma incompleta, entre 1 e $4(46,1 \%)$, em unidades básicas de saúde. Baixa renda e maior número de filhos fragilizaram a realização das consultas $(\mathrm{p}<0,001)$. A imunização e as triagens neonatais mostraram resultados favoráveis; estratificação de risco e a visita domiciliar não aconteceram. A antropometria foi a ação prioritária na puericultura. Conclusão: fatores epidemiológicos e déficits na assistência de enfermagem contribuem para fragilizar a adesão na puericultura.

Palavras-chave: Crescimento; Desenvolvimento infantil; Saúde da criança; Enfermagem pediátrica; Atenção primária à saúde.

\begin{abstract}
Objective: to evaluate actions developed in childcare and factors related to child health follow-up. Method: crosssectional, analytical research, with data collection between 2017 and 2018, with first contact in the maternity hospital, 24 hours after delivery; and in households after six months, with the participation of 280 mothers. For analysis, the Chi-square association test or Fischer's exact test was performed, with a significance level of 5\% (p<0.05). Results: most childcare consultations were carried out by nurses, incompletely, between 1 and 4 (46.1\%), in basic health units. Low income and a greater number of children made the consultation more difficult $(\mathrm{p}<0.001)$. Immunization and neonatal screening have shown favorable results; risk stratification and home visits did not happen. Anthropometry was the priority action in childcare. Conclusion: epidemiological factors and deficits in nursing care contribute to weaken adherence in childcare.Incluir o resumo em inglês.
\end{abstract}

Keywords: Growth; Child development; Child health; Pediatric nursing; Primary health care.

\section{Resumen}

Objetivo: evaluar las acciones desarrolladas en la atención infantil y los factores relacionados con el seguimiento de la salud infantil. Método: investigación transversal, analítica, con recolección de datos entre 2017 y 2018 , con primer contacto en el alojamiento de maternidad, 24 horas después del parto; y en los hogares a los seis meses, con la 
participación de 280 madres. Para el análisis se realizó la prueba de asociación Chi-Cuadrado o prueba exacta de Fischer, con un nivel de significancia del 5\% ( $\mathrm{p}<0.05$ ). Resultados: la mayoría de las consultas de cuidado infantil fueron realizadas por enfermeras, de forma incompleta, entre 1 y $4(46,1 \%)$, en unidades básicas de salud. Los bajos ingresos y un mayor número de hijos dificultan la consulta $(\mathrm{p}<0,001)$. La inmunización y el cribado neonatal han mostrado resultados favorables; la estratificación del riesgo y las visitas domiciliarias no se realizaron. La antropometría fue la acción prioritaria en la atención infantil. Conclusión: los factores epidemiológicos y los déficits en el cuidado de enfermería contribuyen a debilitar la adherencia en el cuidado infantil.

Palabras clave: Crecimiento; Desarrollo infantil; Salud del niño; Enfermería pediátrica; Atención primaria de salud.

\section{Introdução}

A puericultura surgiu no século XIX na Europa e foi criada com o objetivo de oferecer cuidados específicos à criança, para que cresçam e se tornem adultos saudáveis, bem como para desenvolver ações de prevenção e promoção de saúde, representando uma ferramenta de acompanhamento e vigilância à saúde da criança (Silva, Silva \& Figueiredo, 2017).

O termo puericultura refere-se a uma ciência que agrega conhecimentos de fisiologia, higiene, nutrição, sociologia, cultura, desenvolvimento neuropsicomotor e comportamento da criança. Por esta razão, compreende-se que a puericultura tem desempenhado papel essencial para a redução da morbimortalidade infantil (Branquinhos \& Lanza, 2018; Caldeira et al., 2017).

A mortalidade infantil é mais evidente no primeiro ano de vida, em especial no primeiro mês de vida da criança, decorrente de afecções do período perinatal e por Causas Sensíveis a Atenção Primária (Who, 2019; Teixeira et al., 2019; França et al., 2017). As causas de maior frequência de morbimortalidade na atualidade envolvem doenças transmissíveis, afecções perinatais e nutricionais (Teixeira et al., 2019; Souza, Duim \& Nampo, 2019). Doenças comuns na década de 1990 como a diarreia, foram reduzidas substancialmente, considerando os avanços tecnológicos, o acesso em serviços de Atenção Primária à Saúde (APS) e um programa sólido de imunização (França et al., 2017). E muito embora os serviços de APS tenham trabalhado com tecnologias de baixa complexidade, estas apresentam alta efetividade e contribuem sobremaneira na redução das taxas de mortalidade infantil em todo o mundo (Pedraza \& Araújo, 2017).

No âmbito da assistência de enfermagem em serviços de APS há muitos desafios para o cuidado de uma criança pequena, por esse motivo, é fundamental que a equipe de saúde, conheça a realidade de cada família, para assim, delinear a assistência para além das demandas clínicas, mas atender as necessidades sociais e ambientais, por meio do acolhimento e da escuta atentiva (Brígido, Santos \& Prado, 2019; Oliveira \& Souza, 2017). Essas ações envolvem planejamento, organização e preparo das equipes de saúde (Oliveira \& Souza, 2017).

Estudo mostrou que as famílias conhecem e compreendem a importância do seguimento ao crescimento e desenvolvimento infantil, no entanto, deparam-se com dificuldades para aderir à puericultura ocasionada por barreiras institucionais, com relevância ao planejamento das equipes e a (in)flexibilidade para agendamentos das consultas (Rezer, Souza \& Faustino, 2020). Somado as vulnerabilidades programáticas, estudos nacionais e internacionais também descrevem que fatores culturais, econômicos e a desigualdade social interferem sobremaneira na oferta de cuidados à saúde infantil e na adesão às consultas de puericultura (Santos et al., 2019; Lima \& Alves, 2017).

Diante disso, formulou-se a questão norteadora: Quais as ações desenvolvidas nas consultas de puericultura e quais os fatores que contribuem ou fragilizam a sua realização? Deste modo, o objetivo desta pesquisa foi avaliar as ações desenvolvidas na puericultura e os fatores relacionados ao seguimento à saúde infantil.

\section{Metodologia}

Pesquisa transversal, analítica, com abordagem quantitativa, realizada nos municípios da nona Regional de Saúde do Paraná: Foz do Iguaçu, Santa Terezinha de Itaipu, Medianeira, Serranópolis do Iguaçu, Matelândia e Ramilândia. A escolha 
destes ocorreu por sorteio, com exceção de Foz do Iguaçu, por ser o município sede da regional de saúde estudada.

Foi realizado o cálculo amostral da população de cada maternidade que atende gestantes dos referidos municípios, com base no número de partos do ano de 2016, considerando $\mathrm{N}$ tamanho (número de elementos) da população; $\mathrm{n}$ tamanho (número de elementos) da amostra; $\mathrm{n}^{\circ}$ uma primeira aproximação para o tamanho da amostra; E0 erro amostral tolerável (Pito, 2012). Por conhecer o tamanho da população realizou-se o cálculo amostral na seguinte expressão:

$\mathrm{n} 0=1 /(\mathrm{E} 0) 2.0,05=400$

Conhecendo o tamanho da população pode-se corrigir o cálculo anterior por: $\mathrm{n}=\mathrm{N} \cdot \mathrm{n} 0 / \mathrm{N}+\mathrm{n} 0$

Considerou-se uma margem de erro da pesquisa de 5\%, um nível de confiança de 95\%. Definiu-se $10 \%$ como margem de segurança, no entanto, após o primeiro contato com as puérperas realizado nas maternidades, ocorreram perdas ao longo do período de coleta de dados, por mudança de endereço ou número telefônico, sendo que, participaram efetivamente da pesquisa 280 puérperas com seus respectivos bebês, sendo que, após localização da participante não houve recusas.

Foram incluídas puérperas submetidas a partos normais e cesarianas com recém- nascidos vivos. Foram excluídas puérperas com problemas de saúde mental descrito em prontuário e mulheres com impossibilidade da visita domiciliar após três tentativas, por não encontrar o endereço, por mudança de telefone ou ausentes no momento da visita.

A coleta de dados, realizada por acadêmicos do quarto e quinto ano do curso de enfermagem de uma universidade pública, aconteceu nos meses de julho de 2017 a junho de 2018. Os acadêmicos foram previamente treinados para a coleta de dados pelos pesquisadores responsáveis/docentes. Estabeleceu-se o primeiro contato nas maternidades, 24 horas após o nascimento do bebê, no setor de alojamento conjunto; e após seis meses do nascimento da criança, sendo realizadas visitas domiciliares para proceder a coleta de dados.

Para a coleta de dados utilizou-se um instrumento estruturado, o qual foi construído por um grupo de docentes com expertise em saúde materna-infantil, sendo que antes de sua aplicação, foram realizados dois pilotos para testar as variáveis, sendo que, os mesmos não foram considerados para a amostra deste estudo. O referido instrumento contém as seguintes variáveis dependentes: idade; raça/cor; situação conjugal; número de filhos; escolaridade materna; condição de ocupação materna; renda familiar; tipo de parto; paridade; problemas de saúde apresentados pela criança; serviço onde buscou atendimento; estratificação de risco; imunização no hospital e na unidade de saúde (completa ou incompleta para idade); triagem neonatal; busca ativa diante de atrasos vacinais; se não consultou, o motivo; necessidade de hospitalização; motivo da hospitalização; se durante a puericultura recebeu orientações, quais (cuidados coto umbilical, cuidados de higiene, prevenção de acidentes domésticos, vacinação, situações de urgências, etc.); medidas antropométricas verificadas nas consultas; ações durante a puericultura; acompanhamentos através de visita domiciliar; quem realizou as visitas. E as variáveis independentes: consultas de puericultura (completa quando realizou entre 5 e 6 consultas; incompleta entre 1 e 4 consultas; e não realizado). Os municípios inseridos na pesquisa realizam as puericulturas de acordo com as recomendações da Rede Mãe Paranaense (RMP), atual política de atenção à saúde materna e infantil do estado do Paraná, a qual recomenda uma consulta mensal de puericultura até os 12 meses de vida.

Para análise dos dados foi realizado o teste de associação Qui-quadrado ou teste exato de Fischer, e análise de resíduos ajustados. Os testes estatísticos foram realizados no Software SPSS Statistics Base 25.0, com nível de significância de $5 \%(\mathrm{p}<0,05)$.

Este estudo foi submetido e aprovado pelo Comitê de Ética em Pesquisa, sob o parecer $\mathrm{n}^{\circ}$ 2.053.304, CAAE: 67574517.1.1001.5231, e atendeu as normas da Resolução 466/2012, que envolve pesquisas com seres humanos. 


\section{Resultados}

Das 280 crianças participantes, a maioria foi acompanhada até os seis meses de vida por meio de consultas de puericultura, entretanto, foram consideradas incompletas para a idade, entre 1 e 4 consultas.

No tocante às consultas de puericultura e a relação com variáveis sociodemográficas, verificou-se que existe diferença estatística significante entre número de consultas e renda familiar. A análise de resíduos mostra que famílias com renda superior a quatro salários mínimos tendem a levar os filhos a um maior número de consultas, e famílias com renda igual ou menor a um salário mínimo, levam seus filhos com menor frequência. Outras variáveis não mostraram relação estatística com o número de consultas de puericultura, conforme a Tabela 1.

Tabela 1 - Distribuição do número de consultas de puericultura em relação as variáveis sóciodemograficas. Nona Regional de Saúde do Paraná, Brasil, 2019

\begin{tabular}{|c|c|c|c|c|c|c|c|}
\hline \multirow{2}{*}{ Variáveis } & \multicolumn{2}{|c|}{ Adequada } & \multicolumn{2}{|c|}{ Incompleta } & \multicolumn{2}{|c|}{ Não realizou } & \multirow{2}{*}{ Valor $\mathbf{p}$} \\
\hline & $\mathbf{n}$ & $\%$ & $\mathbf{n}$ & $\%$ & $\mathbf{n}$ & $\%$ & \\
\hline \multicolumn{8}{|l|}{ Idade $(\mathrm{n}=\mathbf{2 8 0})$} \\
\hline$\leq 19$ anos & 19 & 42,2 & 24 & 53,3 & 2 & 4,4 & $0,554 *$ \\
\hline 20 a 34 anos & 83 & 42,1 & 89 & 45,2 & 25 & 12,7 & \\
\hline$\geq 35$ anos & 17 & 44,7 & 16 & 42,1 & 5 & 13,2 & \\
\hline \multicolumn{8}{|l|}{ Raça/cor (n=279) } \\
\hline Branca & 68 & 44,7 & 69 & 45,4 & 15 & 9,9 & \\
\hline Negra & 8 & 36,4 & 14 & 63,6 & 0 & 0,0 & \\
\hline Parda & 42 & 40,8 & 44 & 42,7 & 17 & 16,5 & $0,221 \#$ \\
\hline Outra & 1 & 50,0 & 1 & 50,0 & 0 & 0,0 & \\
\hline \multicolumn{8}{|l|}{ Escolaridade $(\mathbf{n}=\mathbf{2 8 0})$} \\
\hline Fundamental completo & 11 & 50,0 & 8 & 36,4 & 3 & 13,6 & \\
\hline Fundamental incompleto & 17 & 28,3 & 32 & 53,3 & 11 & 18,3 & \\
\hline Médio completo & 47 & 51,6 & 53 & 46,9 & 13 & 11,5 & $0,110 \#$ \\
\hline Médio incompleto & 23 & 46,0 & 22 & 44,0 & 5 & 10,0 & \\
\hline Superior completo & 14 & 70,0 & 6 & 30,0 & 0 & 0 & \\
\hline Superior incompleto & 6 & 50,0 & 6 & 50,0 & 0 & 0 & \\
\hline Sem escolaridade & 1 & 33,3 & 2 & 66,7 & 0 & 0 & \\
\hline \multicolumn{8}{|l|}{ Situação conjugal (n=280) } \\
\hline Com companheiro & 107 & 41,6 & 120 & 46,7 & 30 & 11,7 & $0,707 \#$ \\
\hline Sem companheiro & 12 & 52,2 & 9 & 39,1 & 2 & 8,7 & \\
\hline \multicolumn{8}{|c|}{ Ocupação materna $(\mathrm{n}=\mathbf{2 8 0})$} \\
\hline Remunerada & 62 & 46,6 & 56 & 42,1 & 15 & 11,3 & $0,397 *$ \\
\hline Não remunerada & 57 & 38,8 & 73 & 49,7 & 17 & 11,6 & \\
\hline \multicolumn{8}{|l|}{ Renda familiar $(n=280)$} \\
\hline Menos que 1 salário & 14 & 26,9 & 31 & 59,6 & 7 & 13,5 & \\
\hline de 1 a 2 salários & 30 & 41,1 & 36 & 49,3 & 7 & 9,6 & \\
\hline de 2 a 3 salários & 52 & 43,7 & 53 & 44,5 & 14 & 11,8 & $0,031 \#$ \\
\hline 4 ou mais & 20 & 69,0 & 7 & 24,1 & 2 & 6,9 & \\
\hline Não informou & 3 & 42,9 & 2 & 28,6 & 2 & 28,6 & \\
\hline
\end{tabular}

* Teste Qui-quadrado. \# Teste de Fischer. Fonte: Autores.

As consultas de puericultura relacionadas a variáveis obstétricas, como mostra a Tabela 2, não demonstraram diferenças significante entre os tipos de parto, paridade e número de consultas, porém observou-se a existência de diferença estatisticamente significante na relação número de filhos e número de consultas. A análise de resíduos demonstra que as mães que já tem outros filhos, tendem a não realizar as consultas de puericultura em número suficiente para a faixa etária. 
Tabela 2 - Distribuição do número de consultas de puericultura em relação as variáveis obstétricas. Nona Regional de Saúde do Paraná, Brasil, 2019.

\begin{tabular}{|c|c|c|c|c|c|c|c|}
\hline \multirow{2}{*}{ Variáveis } & \multicolumn{2}{|c|}{ Adequada } & \multicolumn{2}{|c|}{ Incompleta } & \multicolumn{2}{|c|}{ Não realizou } & \multirow[t]{2}{*}{ Valor p } \\
\hline & $\mathbf{n}$ & $\%$ & $\mathbf{n}$ & $\%$ & $\mathbf{n}$ & $\%$ & \\
\hline \multicolumn{8}{|c|}{ Tipo de parto $(n=272)$} \\
\hline Normal & 58 & 41,4 & 68 & 48,6 & 14 & 10,0 & $0,532 *$ \\
\hline Cesáreo & 57 & 43,2 & 57 & 43,2 & 18 & 13,6 & \\
\hline \multicolumn{8}{|c|}{ Tem filho $(n=280)$} \\
\hline Sim & 61 & 37,6 & 74 & 45,7 & 27 & 16,7 & $0,001 *$ \\
\hline Não & 58 & 49,1 & 75 & 63,6 & 5 & 4,2 & \\
\hline \multicolumn{8}{|c|}{ Paridade $(n=280)$} \\
\hline $1^{\mathrm{a}}$ & 49 & 46,2 & 42 & 39,6 & 15 & 14,1 & \\
\hline $2^{\mathrm{a}}$ & 37 & 45,5 & 41 & 47,1 & 9 & 10,3 & \\
\hline $3^{a}$ & 16 & 32,6 & 28 & 57,1 & 5 & 10,2 & $0,562 \#$ \\
\hline $4^{\mathrm{a}}$ ou maior & 17 & 44,7 & 18 & 47,4 & 3 & 7,9 & \\
\hline
\end{tabular}

* Teste Qui-quadrado. \# Teste de Fischer. Fonte: Autores.

No que se refere às ações de promoção da saúde e prevenção de doenças, existe diferença significante entre as proporções, conforme a tabela 3. Para as participantes, o serviço de saúde de primeira escolha para o atendimento de saúde de rotina do filho é a Unidade Básica de Saúde (UBS), e o profissional que realiza o acompanhamento ao crescimento e desenvolvimento da criança é o enfermeiro.

No tocante a imunização da criança no ambiente hospitalar e em serviços de APS, bem como, a realização das triagens neonatais (Teste do Olhinho, Orelhinha, Pezinho e Coraçãozinho) os resultados mostraram-se satisfatórios. 
Tabela 3 - Ações de promoção da saúde e prevenção de doenças para a criança com menos de seis meses de vida. Nona Regional de Saúde do Paraná, Brasil, 2019.

\begin{tabular}{|c|c|c|c|c|}
\hline Variáveis & & $\mathbf{n}$ & $\%$ & Valor p \\
\hline \multirow[t]{3}{*}{ Consultas de puericultura $(n=280)$} & 1 a 4 & 129 & 46,1 & \\
\hline & 5 ou mais & 119 & 42,6 & 0,001 \\
\hline & Não realizou & 32 & 11,4 & \\
\hline \multirow[t]{6}{*}{ Serviço de primeira escolha $(n=123)$} & UBS & 187 & 66,8 & \\
\hline & UBS + UPA & 20 & 7,1 & \\
\hline & UBS + consultório particular & 3 & 1,1 & 0,001 \\
\hline & UPA & 56 & 20,0 & \\
\hline & Consultório particular & 10 & 3,6 & \\
\hline & Outro & 4 & 1,4 & \\
\hline \multirow[t]{8}{*}{ Profissional nas consultas $(n=261)$} & Enfermeiro & 65 & 24,9 & \\
\hline & Enfermeiro + pediatra & 61 & 23,4 & \\
\hline & Enfermeiro + médico da família & 27 & 10,3 & 0,001 \\
\hline & Enfermeiro + pediatra + médico & 21 & & \\
\hline & da família & & 8,0 & \\
\hline & Médico pediatra e da família & 13 & 5,0 & \\
\hline & Médico pediatra & 60 & 23,0 & \\
\hline & Médico da família & 14 & 5,4 & \\
\hline \multirow[t]{2}{*}{ Estratificação de risco $(n=280)$} & Realizada & 5 & 1,8 & 0,001 \\
\hline & Não realizada & 275 & 98,2 & \\
\hline \multirow[t]{2}{*}{ Visitas domiciliares $(n=280)$} & Sim & 89 & 31,8 & 0,001 \\
\hline & Não & 191 & 61,2 & \\
\hline \multirow[t]{3}{*}{ Triagem neonatal $(n=280)$} & Adequada & 278 & 99,3 & 0,001 \\
\hline & Incompleta & 2 & 0,7 & \\
\hline & Não realizada & 0 & 0 & \\
\hline \multirow[t]{3}{*}{ Imunização no hospital (n=280) } & Completa & 280 & 100,0 & \\
\hline & Incompleta & 0 & 0 & \\
\hline & Não realizada & 0 & 0 & \\
\hline \multirow[t]{3}{*}{ Imunização nos serviços de APS $(\mathbf{n}=280)$} & Completa & 255 & 91,0 & 0,001 \\
\hline & Incompleta & 24 & 8,6 & \\
\hline & Não realizada & 1 & 0,4 & \\
\hline
\end{tabular}

* Teste Qui-quadrado (proporções esperadas iguais).

Legenda: Unidade Básica de Saúde (UBS); Unidade de Pronto Atendimento (UPA); Atenção Primária à Saúde (APS). Fonte: Autores.

Com respeito às ações de cuidado à saúde da criança, verificou-se pela tabela 4 , que embora a maioria das crianças não apresentaram problemas de saúde após o primeiro mês de vida, destaca-se que aproximadamente $40 \%$ das mães descreveram algum tipo de problema com o filho, principalmente de origem respiratória, os quais mostraram significância estatística.

O serviço de saúde de primeira escolha para as mães com os filhos doentes foi a Unidade de Pronto Atendimento (UPA), seguida dos serviços de APS. A hospitalização foi necessária para 28,7\% das crianças que precisaram de serviços de saúde, e problemas respiratórios foram os diagnósticos de internação de maior frequência (Tabela 4). 
Tabela 4 - Problemas de saúde e necessidade de hospitalização de crianças menores de seis meses de vida. Nona Regional de Saúde do Paraná, Brasil, 2019.

\begin{tabular}{|c|c|c|c|c|}
\hline Variáveis & & $\mathbf{n}$ & $\%$ & Valor p \\
\hline \multirow[t]{2}{*}{ Problemas de saúde $(\mathrm{n}=280)$} & Sim & 123 & 43,9 & 0,048 \\
\hline & Não & 157 & 56,1 & \\
\hline \multirow[t]{8}{*}{ Problemas de saúde (n=133) } & Respiratórios & 84 & 63,2 & \\
\hline & Gastrointestinais & 8 & 6,0 & \\
\hline & Cardiovascular & 3 & 2,3 & \\
\hline & Geniturinários & 16 & 12,0 & 0,001 \\
\hline & Cirúrgicos & 4 & 3,0 & \\
\hline & Hemorrágicos & 3 & 2,3 & \\
\hline & Alergias & 3 & 2,3 & \\
\hline & Outros & 12 & 9,0 & \\
\hline \multirow[t]{5}{*}{ Serviço procurado $(n=280)$} & UPA & 62 & 22,1 & \\
\hline & UBS ou USF & 44 & 15,7 & \\
\hline & Consulta particular & 10 & 3,6 & 0,001 \\
\hline & Outros & 7 & 2,5 & \\
\hline & Não procurou & 157 & 56,1 & \\
\hline \multirow[t]{2}{*}{ Internação hospitalar $(n=123)$} & Sim & 35 & 28,7 & 0,001 \\
\hline & Não & 88 & 72,1 & \\
\hline \multirow[t]{6}{*}{ Motivo da internação $(n=35)$} & Alterações respiratórias & 16 & 45,7 & \\
\hline & Necessidade de cirurgia & 4 & 11,4 & \\
\hline & Gastrointestinal & 4 & 11,4 & 0,001 \\
\hline & Icterícia & 3 & 8,6 & \\
\hline & Infecções & 3 & 8,6 & \\
\hline & Outras & 5 & 14,3 & \\
\hline
\end{tabular}

De acordo com a Figura 1, a prioridade nas consultas de puericultura foram à mensuração dos dados antropométricos (peso, estatura, perímetro cefálico e torácico), que se referem ao acompanhamento do crescimento infantil. Outras ações como o exame físico, aferição de sinais vitais e avaliação da amamentação foram observadas em menores proporções.

Sobre as orientações, a imunização, aleitamento materno e introdução alimentar tem sido as prioridades para os profissionais de saúde. Orientações importantes foram reportadas por metade das mães, como cuidados com o coto umbilical e situações de emergências, todavia, informações relativas a prevenção de acidentes domésticos e cuidados de higiene foram descritas por aproximadamente $20 \%$ das participantes. 
Figura 1 - Práticas de saúde e orientações realizadas durante a consulta de puericultura de crianças com menos de seis meses de vida. Nona Regional de Saúde do Paraná, Brasil, 2019.



Fonte: Autores.

\section{Discussão}

Os resultados da presente pesquisa mostraram que a realização de consultas de puericultura encontra-se inadequada de acordo com o preconizado pela RMP, atual política de saúde materno e infantil do estado Paraná, a qual determina que até o sexto mês da criança, é preciso realizar seis consultas de puericultura (Sesa/PR, 2018; Caldeira et al., 2017). Foram verificados que fatores sociodemográficos e obstétricos podem ter fragilizado o seguimento ao crescimento e desenvolvimento da criança pelos serviços de APS na região estudada.

Fatores como idade materna, raça/cor, escolaridade, situação conjugal e ocupação materna não foram relevantes para potencializar ou fragilizar as consultas de puericultura, entretanto, a variável renda mostrou-se significativa com relação ao número de consultas de puericultura, inferindo que, famílias com renda menor ou igual a um salário mínimo levam menos os filhos para a puericultura, quando comparado a famílias com renda superior a quatro salários mínimos. Este resultado foi evidenciado por outro estudo, que revelou que a renda familiar é considerada um fator condicionante para o acompanhamento da criança, ou seja, quanto menor a renda familiar, menor a adesão ao seguimento nas puericulturas (Cordeiro et al., 2019).

A literatura científica traz que a renda apresenta estreita ligação com o comportamento das mães sobre as práticas de promoção da saúde do filho (Silva, Silva \& Figueiredo, 2017). A situação financeira potencializa a qualidade de vida, sendo a saúde seu principal resultante, visto que direciona a tomada de decisão familiar quanto sua prioridade, gerando assim, prejuízos nos cuidados à criança. Importante destacar que outros aspectos também podem interferir no seguimento da saúde da criança, como exemplo, às condições de moradia, alimentação e higiene (Silva, Silva \& Figueiredo, 2017).

Com respeito ao histórico obstétrico materno, a relação entre mães com mais de um filho e o menor número de consultas de puericultura, demonstrou que algumas vezes, por já apresentar conhecimento prévio em relação as ações desenvolvidas na puericultura, as mães consideram não haver a necessidade de realizar os mesmos passos, uma vez que isto já foi feito em sua primeira maternidade. Resultado diferente foi mostrado no estudo realizado em Jaboatão dos Guararapes no Estado de Pernambuco, onde as mães com mais de um filho buscaram manter em dia a puericultura e a imunização dos filhos menores (Cordeiro et al., 2019).

Ademais, promover saúde não se refere apenas a um modo de vida saudável, trata-se de uma responsabilidade do setor da saúde e sociedade como um todo. Diante de fragilidades como as citadas, cabe à equipe assistencial gerar meios para que assistência seja proporcional às necessidades familiares de momento, cabendo ao enfermeiro à responsabilidade técnicacientífica de monitorar, avaliar e intervir no processo saúde-doença para o segmento infantil (Goes et al., 2018). A puericultura colabora com estratégias para promover saúde, pois propicia o acompanhamento efetivo de mãe e filho, a partir de trocas de 
experiências e apoio as dificuldades (Cordeiro et al., 2019).

Quanto ao número de consultas de puericultura, o estudo realizado no interior do Rio Grande do Sul, revelou que os pais faltosos justificam o seu não comparecimento às consultas devido aos horários de trabalho e por já realizarem consultas médicas anteriores (Merg \& Menezes, 2018). Logo, é possível inferir que existe a falta de informação às famílias, que resulta na manutenção de uma cultura curativista e centrada na figura médica, fragmentando ações oportunas de promoção da saúde por meio do seguimento ao crescimento e desenvolvimento infantil (Merg \& Menezes, 2018).

Deste modo, torna-se extremamente importante que o enfermeiro realize ações educativas e as leve até a comunidade, explicando sobre a necessidade e importância das consultas de puericultura para a vigilância da saúde infantil. Para isso, o enfermeiro precisa lançar mão de ferramentas estratégicas como a visita domiciliar para buscar faltosos e identificar problemas que levam à baixa adesão às consultas (Sesa/PR, 2018).

A literatura destaca que a diversidade de ações dispostas nos serviços de APS, como a imunização e o seguimento do crescimento e desenvolvimento infantil, reduz de forma efetiva o número de hospitalizações por causas evitáveis. Para isso, os profissionais de saúde não devem se limitar à avaliação do crescimento durante as consultas de puericultura, mas devem compreender que este se trata de um momento oportuno para relacionar as práticas de cuidar com o contexto domiciliar e com as experiências familiares para promover saúde (Viera et al., 2018).

Nesta pesquisa foi identificado que o primeiro serviço procurado pelas mães para a atenção à saúde do filho foi o serviço de APS e o enfermeiro, o protagonista no desenvolvimento das atividades de puericultura. Os serviços de APS devem ser considerados a porta de entrada para o sistema de saúde, organizados por meio de ações programáticas, com atendimentos às maiores necessidades de saúde, envolvendo promoção e regulação do acesso à outros níveis de atenção, garantindo integralidade e continuidade da atenção à saúde (Arantes, Shimuzu \& Merchán-Hamann, 2016).

Quanto à busca por atendimento ao filho em situação de adoecimento, as mães participantes recorreram primeiramente as UPA, considerando ser um serviço de atendimento rápido e a qualquer horário quando necessário, pois os serviços de APS da região em estudo, funcionam com horários e dias limitados, tornando-se restrito ao atendimento às demandas de agendamento de consultas (Zanardo et al., 2017).

As ações de promoção à saúde, prevenção de doenças e às condições crônicas nos serviços de APS contemplam um trabalho com visão vertical de programas e protocolos, um modelo enrijecido e segmentado dentro das Redes de Atenção à Saúde (RAS). Neste modelo de organização seletiva e de grupos prioritários, a população sintomática na maioria recorre em primeira escolha para as UPA, para situações agudas e crônicas (Vieira et al., 2019; Ros et al., 2018).

Somada a essas ações, a estratificação de risco garante a criança cuidado adequado e oportuno, pois através das ações programáticas, as situações que envolvem fatores clínicos complexos, com risco real ou fatores preveníveis que demandem intervenções com maiores rearranjos tecnológicas a criança deverá ser necessariamente referenciada, porém ainda acompanhada pelos serviços de APS (Sesa/PR, 2018). A negligência na realização da estratificação de risco, ou mesmo a falta de registro na carteira de saúde, como evidenciado na presente pesquisa, impossibilita que a criança receba os cuidados adequados a sua condição atual (Brígido, Santos \& Prado, 2019).

Do mesmo modo, a visita domiciliar pouco evidenciada neste estudo, refere-se a uma ferramenta de vigilância a saúde infantil. Promove um olhar diferenciado ao processo saúde e doença, por meio do encontro entre profissional e famílias, proporcionando cuidados em domicílio, com mais conforto, visto que, oportuniza a observação das reais condições de vida da família (Goes et al., 2018). Pela visita domiciliar o profissional pode identificar as necessidades familiares, para em seguida, elaborar um plano assistencial voltado para a realidade em qual vivem (Luz et al., 2016).

No tocante as triagens neonatais, os resultados deste estudo mostraram-se satisfatórios. Mas muito além da realização destes testes, é fundamental que os profissionais repassem informações consistentes e claras sobre seus resultados, pois estas 
ações podem aumentar a compreensão da família sobre a necessidade da triagem neonatal, e do acompanhamento, caso algum resultado não demonstre padrões de anormalidade (Oliveira \& Souza, 2017).

Com respeito a imunização infantil no ambiente hospitalar e nos serviços de APS, verificou-se que esta é uma importante ação para garantir saúde e evitar a morbimortalidade infantil e tem alcançado resultados favoráveis no cenário estudado (Vieira et al., 2018). A cobertura vacinal na UBS esteve um pouco abaixo do recomendado com 91\%, pois pelo Ministério da Saúde, desde 1990, há uma recomendação acima de 95\%. Entretanto, desde o ano de 2016, há evidências de redução da cobertura vacinal, fator preocupante, pois esta redução poderá aumentar os índices de mortalidade infantil (Sato, 2018). Exemplo disso é o retorno de doenças erradicadas como o sarampo, diagnosticado em muitos estados brasileiros (Sato, 2018).

Sobre o adoecimento das crianças com menos de seis meses, dentre os problemas identificados, os respiratórios ocorreram com maior intensidade. Alterações respiratórias ocorrem com maior frequência em comparação aos outros problemas de saúde e representa uma causa importante para internações hospitalares, mesmo tratando-se de uma doença prevenível e tratável com tecnologias de baixo custo, disponíveis em serviços de APS (Zanardo et al., 2017; Pedraz \& Araujo, 2017).

O acompanhamento planejado da criança, em comum às ações de monitoramento das doenças, como afecções respiratórias agudas e diarreias, bem como, o estímulo ao aleitamento materno, imunização e orientação alimentar, contribui sobremaneira na promoção, qualidade de vida e crescimento e desenvolvimento saudável, ações estas que realizadas corretamente poderiam ter evitado o número de crianças hospitalizadas na região em estudo (Branquinhos \& Lanza, 2018).

Com relação às ações durante as consultas de puericultura, os dados antropométricos foram os mais realizados, ação na qual o enfermeiro detecta alterações no crescimento e desenvolvimento da criança, requerendo avaliações e encaminhamentos para especialidades, sempre que necessário (Zanardo \& Andra, 2017). Dados semelhantes a estes foram identificados em um município de médio porte do norte do Paraná, que demonstrou que os dados antropométricos sobressaem diante de outras ações, com cerca de 100\% de registro nos prontuários durante as puericultura (Stalin, André \& Gozi, 2019).

Outra ação importante a ser realizada durante as consultas de seguimento envolve o exame físico, pois através desta ação é possível identificar sinais e sintomas indesejáveis, avaliá-los e oportunizar precocemente o tratamento adequado para cada problema (Zanardo \& Andrade, 2017).

Além disso, a literatura científica destaca que o enfermeiro não deve limitar-se apenas aos afazeres técnicos na puericultura, pois devem fornecer orientações à família sobre questões referente aos cuidados e à saúde da criança (Goes et al., 2018). As orientações incluem os cuidados gerais com a criança, higiene, imunização, aleitamento materno, alimentação infantil, prevenção de acidentes, comportamento familiar, e importância da avaliação constante da puericultura (Sesa/PR, 2018). Operacionalizar sistematicamente as consultas de puericultura possibilitará a qualificação do processo assistencial e fortalecerá a integralidade da atenção à saúde da criança no âmbito da APS (Siega et al., 2020).

Os resultados desta pesquisa demonstraram-se insipientes com relação às orientações para estes cuidados. Estudos enfatizam que a prática educativa antes de tudo, necessita de vínculo, confiança e comunicação efetiva entre enfermeiros e famílias (Stalin, André \& Gozi, 2019; Zanardo \& Andrade, 2017). A falta de orientações adequadas durante a puericultura dificulta o esclarecimento e a importância que o acompanhamento da saúde da criança representa.

A pesquisa apresentou como limitação a necessidade de dar voz aos profissionais de saúde dos serviços de APS para que possam apontar fatores determinantes para contemplar a integralidade das ações em saúde na primeira infância. Mas de todo modo, a pesquisa possibilitou mostrar aspectos importantes para as ações do cuidado infantil, e demonstrou a importância do enfermeiro, como protagonista e ordenador do seguimento ao crescimento e desenvolvimento infantil. 


\section{Conclusão}

A maioria das crianças realizou as consultas de puericultura, contudo, foram realizadas de forma incompleta, entre $1 \mathrm{e}$ 4 consultas, por enfermeiros em unidades básicas de saúde. A baixa renda familiar e o maior número de filhos foram identificados como fatores que podem fragilizar a realização das consultas. Ações para a promoção da saúde na primeira infância como a imunização e as triagens neonatais mostraram resultados favoráveis, todavia, a estratificação da criança de risco e a visita domiciliar não foram contempladas neste estudo. Sobre as ações recomendadas na puericultura, o enfoque esteve centrado na aferição das medidas antropométricas e não contemplaram a integralidade da atenção para promoção de saúde e prevenção de agravos.

Esta pesquisa buscou descrever como estão organizados os cuidados de saúde da criança nos primeiros seis meses de vida, para assim, subsidiar ações para aprimorar o seguimento ao crescimento e desenvolvimento na primeira infância. Contudo, a pesquisa mostrou fatores epidemiológicos que quando agregados ao déficit na assistência, seja pelos profissionais de saúde ou mesmo por falhas do sistema operacional, resultam na diminuição da adesão do acompanhamento do crescimento e desenvolvimento da criança. É preciso avançar nas ações de promoção e prevenção, para assim identificar problemas precocemente e evitar a hospitalização na primeira infância por causas evitáveis.

\section{Referências}

Arantes, L. J., Shimizu, H. E. , \& Merchán-Hamann, E. (2016). Contribuições e desafios da estratégia Saúde da família na atenção primária à saúde no Brasil: revisão da literatura. Ciência \& Saúde Coletiva, 21 (5), 1499-1509.

Branquinhos, I. D., \& Lanza, F. M. (2018). Saúde da criança na atenção primária: evolução das políticas brasileiras e a atuação do enfermeiro. Revista de Enfermagem do Centro-Oeste Mineiro, 8, 2753.

Brígido, A. F., Santos, E. O., \& Prado, E. V. (2019). Qualificação do cuidado a puericultura: uma intervenção em serviço na estratégia de saúde da família. Revista de Pesquisa Cuidado é Fundamental Online, 11 (esp), 448-454.

Caldeira, S., Luz, M. S., Tacla, M. T. G. M., Machineski, G. G., Silva, R. M. M., Pinto, M. P. V., \& Ferrari, R. A. P. (2017). Ações de cuidado do enfermeiro no programa rede mãe paranaense. Revista Mineira de Enfermagem, 21, e992.

Cordeiro, E. L., Silva, L. S. R., Urquiza, J. L., Nascimento, M. A., Silva, R. M., Souza, G. C. S., Douberin, C. A., Pimenta, C. S., \& Mendes Filho, E. B. (2019). Conhecimento das mães sobre o esquema vacinal de seus filhos assistidos em uma unidade básica de saúde. Brazilian Journal of Health Review, 2 (1), 644-660.

França, E. B., Lanski, S., Rego, M. A. S., Malta, D. C., França, J. S., Teixeira, R., Porto, D., Almeida, M. F., Souza, M. F. M., Szwarcwald, C. L., Mooney, M., Naghavi, M., \& Vasconcelos, A. M. N. (2017). Principais causas da mortalidade na infância no Brasil, em 1990 e 2015: estimativas do estudo de carga global de doença. Revista Brasileira de Epidemiologia, 20 (Suppl1), 46-60.

Góes, F. G. B., Silva, M. A., Paula, G. K., Oliveira, L. P. M., Mello, N. C., \& Silveira, S. S. D. (2018). Contribuições do enfermeiro para boas práticas na puericultura: revisão integrativa da literatura. Revista Brasileira de Enfermagem, 71 (Suppl6), 2808-2817.

Lima, B. S., \& Alves, F. Q. (2017). Fatores sócio-culturais e económicos que interferem na adesão a consulta de puericultura: relato de experiencia. Revista das Semanas Acadêmicas da ULBRA Cachoeira do Sul, 4 (6), 1-15.

Luz, V. L. E. S., Sales, J. C. S., Siqueira, M. L. S., Vieira, T. S., Coêlho, D. M. M., \& Barbosa, M. G. (2016). Assistência do enfermeiro da estratégia saúde da família na visita domiciliar à puérpera. Revista Interdisciplinar, 9 (1), 13-23.

Merg, C. C. K., \& Menezes, L. P. (2018). A implantação da consulta de enfermagem em puericultura na estratégia de saúde da família. Revista Espaço Ciências \& Saúde, 6 (1), 41-55.

Oliveira, E. F., Souza, A. P. A. (2017). Importância da realização precoce do teste do pezinho: o papel do enfermeiro na orientação da triagem neonatal. Revista Multidisciplinar de Psicologia, 11 (35), 362-78.

Pedraza, D. F., \& Araújo, E. M. N. (2017). Internações das crianças brasileiras menores de cinco anos: revisão sistemática da literatura. Revista de Epidemiologia e Serviços de Saúde, 26, (1), 169-182.

Pito, A. L. B. S. (2012). Epidemiologia aplicada nos serviços de saúde. Editora Martinari.

Rezer, F., Souza, T. V., \& Faustino, W. R. (2020). Dificuldades dos responsáveis por crianças na adesão a puericultura. Journal Health NPEPS, 5 (1), 338350 .

Ros, C., Peres, A. M., Bernardino, E., Lowen, I. M. V., Poli Neto, P., \& Moura, M. E. B. (2018). Atenção primária à saúde: ordenadora da integração assistencial na rede de urgência e emergência. Revista Mineira de Enfermagem, 22, e1137. 
Research, Society and Development, v. 10, n. 6, e53710616048, 2021

(CC BY 4.0) | ISSN 2525-3409 | DOI: http://dx.doi.org/10.33448/rsd-v10i6.16048

Santos, A. M. A., Perelman, J., Jacinto, P. A., Tejada, C. A. O., Barros, A. J. D., Bertoldi, A. D., Matijasevich, A., \& Santos I. A. (2019). Income-related inequality and inequity in child health care: a longitudinal analysis using data from Brazil. Social Science \& Medicine, 224, 127-137.

Sato, A. P. S. (2018). Qual a importância da hesitação vacinal na queda das coberturas vacinais no Brasil? Revista de Saúde Pública, 52 (96), 1-9.

Secretaria de Estado da Saúde do Paraná (2018). Programa Rede Mãe Paranaense. Linha guia. SESA-PR: SESA-PR.

Siega, C. K., Adamy, E. K., Toso, B. R. G. O., Zocche, D. A. A., \& Zanatta, E. A. (2020). Vivências e significados da consulta do enfermeiro em puericultura: análise à luz de Wanda Horta. Revista de Enfermagem UFSM, 10 (e65), 1-21.

Silva, D. M., Silva, J. G. V., \& Figueiredo, C. A. R. (2017). Assistência de enfermagem em puericultura: um estudo bibliográfico. Revista Saber Científico, 6 (1), 46-60.

Souza, S., Duim, E., \& Nampo, F. K. (2019). Determinants of neonatal mortality in the largest international border of Brazil: a case-control study. BMC Public Health, 19, 1304.

Stalin, R. R. P., André, N. J., \& Gozi, T. M. B. (2019). Perfil das consultas de puericultura realizadas somente por enfermeiros. Revista Terra \& Cultura: Cadernos de Ensino e Pesquisa, 35 (esp), 93-106.

Teixeira, J. A. M., Araújo, W. R. M., Maranhão, A. G. K., Cortez-Escalante, J. J., Rezende, L. F. M., \& Matijasevich, A. (2019). Mortalidade no primeiro dia de vida: tendências, causas de óbito e evitabilidade em oito unidades da federação brasileira, entre 2010 e 2015 . Revista de Epidemiologia e Serviços de Saúde, 28 (1), e2018132.

Unicef, Who. Levels \& trends in child mortality. Report (2019). https://data.unicef.org/resources/levels-and-trends-in-child-mortality/

Vieira, D. S., Dias, T. K. C., Pedrosa, P. K. B., Vaz, E. M. C., Collet, N., \& Reichert, A. P. S. (2019). Processo de trabalho de enfermeiros na vigilância do desenvolvimento infantil. Revista Mineira de Enfermagem, 23, e-1242.

Vieira, D. S., Santos, N. C. C. B., Nascimento, J. A., Collet, N., Toso, B. R. G. O., \& Reichert, A. P. S. (2018). A prática do enfermeiro na consulta de puericultura na estratégia saúde da família. Texto \& Contexto de Enfermagem, 27 (4), e4890017:2-10.

Zanardo, G. M., Andrade, U., Zanardo, G. M., \& Menezes, L. P. (2017). Atuação do enfermeiro na consulta de puericultura: uma revisão narrativa da literatura. Revista Enfermagem, 13 (13), 55-69. 